\title{
Role of Physical Mechanisms in Biological Self-Organization
}

\author{
Adrian Neagu, ${ }^{1,2}$ Karoly Jakab, ${ }^{1}$ Richard Jamison, ${ }^{1,3}$ and Gabor Forgacs ${ }^{1,4}$ \\ ${ }^{1}$ Department of Physics, University of Missouri, Columbia, Missouri 65211, USA \\ ${ }^{2}$ Department of Biophysics and Medical Informatics, Victor Babes University of Medicine and Pharmacy, 1900 Timisoara, Romania \\ ${ }^{3}$ Department of Electrical Engineering, Clemson University, Clemson, South Carolina 29634, USA \\ ${ }^{4}$ Department of Biology, University of Missouri, Columbia, Missouri 65211, USA
}

(Received 6 December 2004; published 21 October 2005)

\begin{abstract}
Organs form during morphogenesis, the process that gives rise to specialized biological structures of specific shape and function in early embryonic development. Morphogenesis is under strict genetic control, but shape evolution itself is a physical process. Here we report the results of experimental and modeling biophysical studies on in vitro biological structure formation. Experimentally, by controlling the interaction between cells and their embedding matrices, we were able to build living structures of definite geometry. The experimentally observed shape evolution was reproduced by Monte Carlo simulations, which also shed light on the biophysical basis of the process. Our work suggests a novel way to engineer biological structures of controlled shape.
\end{abstract}

DOI: 10.1103/PhysRevLett.95.178104

Embryonic tissues go through complex patterning, called morphogenesis, in the course of which the organism and its parts gradually develop their final shape. Morphogenesis is a self-assembly process which leads to complex structures (i.e., organs) starting from simpler interacting components (i.e., cells) [1]; it is under strict genetic control. However, genes do not produce forms and shapes, physical mechanisms do [2,3]. The biochemical processes associated with gene activity thus must set up the permissive conditions for the physical mechanisms, which in turn constrain the outcome of biological structure formation.

One physical mechanism long known and amply demonstrated to act in morphogenetic processes is based on the apparent similarity between liquids and tissues composed of motile and adhesive cells (e.g., most embryonic tissues) $[4,5]$. Tissue fragments of such cells round up into spheres to minimize their interfacial area [6,7]. Contiguously placed spheres fuse with the same kinetics as liquid drops [8]. Two randomly intermixed distinct cell populations sort, with the same time evolution and final configuration as phase separating immiscible liquids [9]. All these phenomena can be interpreted in terms of tissue interfacial tensions and viscosities, which have been measured for a number of cell types and their values found to be consistent with the mutual sorting behavior of the corresponding tissues [6,7,10-14].

The molecular basis of tissue liquidity has been established by Steinberg, who formulated the differential adhesion hypothesis [4], stipulating that it is the distinct cell adhesion apparatus characterizing cohesive tissues that gives rise to their surface tensions [5]. Despite confirmation through in vivo experiments [15-17] and computer simulations $[18,19]$, the notion of tissue liquidity remains puzzling. The movement of loosely bound liquid particles is driven by van der Waals forces and powered by thermal energy whose scale is set by $k_{B} T\left(k_{B}\right.$ is the Boltzmann
PACS numbers: 87.17.-d, 02.70.Uu, 87.18.La, 89.75.Fb

constant and $T$ the absolute temperature). The motion of cells bound in tissues by cell adhesion molecules is powered by metabolic energy, whose scale is set by adenosine triphosphate hydrolysis. Thus, it is the analogy (as opposed to identity) between liquids and tissues, together with the known physics of the former, that provides useful information for the latter.

We have shown earlier that tissue liquidity can be employed to engineer 3D living structures of specific shape [20]. In particular, we used spheroidal cell aggregates, as discrete units, to build simple toroidal (or short tubular) cellular structures. This was accomplished by placing the aggregates along a circle into hydrogels (i.e., scaffolds). Structure formation took place by the subsequent fusion of the aggregates. Computer simulations of the same process based on the liquidlike properties of these aggregates fully reproduced the experimental results. These findings raise the questions of whether the liquid analogy can be used to fabricate anatomical structures and what useful information our model can provide to such an endeavor.

Here we show that tissue liquidity could provide the scientific basis of a novel type of tissue engineering that uses bioprinting [21]. We constructed model spherical cell aggregates (i.e., "bioink" particles) and deposited (i.e., "printed") them into model matrices (i.e., "biopaper"). The outcome of postprinting structure formation depended on the properties of the biopaper. Under permissive conditions we simulated the formation of "physiological" structures: lumenized tubes and thick cellular sheets. Lumenized tubes may represent blood vessels; cellular sheets (depending on cell type) could be used as skin or cardiac grafts. Sheets were also produced experimentally by printing spherical aggregates of Chinese hamster ovary (CHO) cells genetically manipulated to express $N$ cadherins (a particular type of cell adhesion molecules) into scaffolds made of agarose or collagen 
type 1 (an essential component of the extracellular milieu of living systems).

Inspired by earlier computer simulations of cell sorting $[18,19]$, we modeled the tissue-gel system on a cubic lattice. We associated with each site of the lattice a spin variable $\sigma$, which accounts for the occupancy of the site by either a gel particle $(\sigma=0)$ or a cell $(\sigma=1)$. The interaction energy of the system is given by $E=$ $\sum_{\left\langle r, r^{\prime}\right\rangle} J\left(\sigma_{r}, \sigma_{r^{\prime}}\right)$, where $r$ and $r^{\prime}$ label lattice nodes and the sum runs over first, second, and third nearest neighbors. The terms in the above sum may take either of the values $J(0,0)=-\varepsilon_{g g}, \quad J(1,1)=-\varepsilon_{c c}, \quad$ or $J(1,0)=J(0,1)=$ $-\varepsilon_{c g}$, where the $\varepsilon$ 's are positive parameters accounting for the strengths of gel-gel, cell-cell, and cell-gel interactions, respectively. (It is assumed that a cell interacts to the same extent with all the neighbors it comes in contact with.) Separating interfacial and bulk terms in the energy, we obtain (up to an irrelevant additive constant) $E=$ $\gamma_{c g} N_{c g}$, where $N_{c g}$ stands for the total number of cell-gel bonds and $\gamma_{c g}=\left(\varepsilon_{c c}+\varepsilon_{g g}\right) / 2-\varepsilon_{c g}$ is proportional to the cell-gel interfacial tension [22].

We simulated structure formation by the Monte Carlo method using the Metropolis algorithm [23]. During a Monte Carlo step (MCS), each cell from the aggregategel interface had the opportunity to move once, exchanging its position with a neighboring gel particle chosen by chance. Cells were constrained to move within the gel. The energy change, $\Delta E$, corresponding to each move was calculated and the new configuration accepted with a probability $P=1$ if $\Delta E \leq 0$ or $P=\exp (-\beta \Delta E)$ if $\Delta E>$ 0 . Here $\beta=1 / E_{T}$ is a measure of the spontaneous, cytoskeleton-driven motion of cells. The average biological fluctuation energy of a cell, $E_{T}$, was shown to be analogous to the thermal energy, $k_{B} T$, of true liquid molecules [24]. Its value depends on cell type and has been estimated for certain embryonic chicken cells [9].

We first built cellular sheets both in silico and in vitro. In case of real cells we printed $25 \mathrm{CHO}$ cell aggregates into various biopapers (i.e., biocompatible gels) either in a square or hexagonal arrangement using the bioprinter in Fig. 1. (For the preparation and handling of the spherical cell aggregates, see Ref. [7] or Ref. [10].) As was shown earlier [20], structure formation depends on the properties of the biopaper. In agarose (known to represent nonadhesive cellular environment and thus serving as control) no fusion of the aggregates took place, a situation reproduced in the simulations with large $\gamma_{c g} / E_{T}$ (results not shown). In case of collagen, upon increasing its concentration (decreasing $\gamma_{c g} / E_{T}$ in the simulations), the gel became progressively more "permissive," eventually facilitating the rapid dispersion of the cells into the matrix (as shown in Ref. [20]). Figure 2 presents the results of simulations and printing for $1.0 \mathrm{mg} / \mathrm{ml}$ collagen concentration, when sheetlike cellular structures formed. Cell viability at the end of each fusion experiment was checked (with trypan

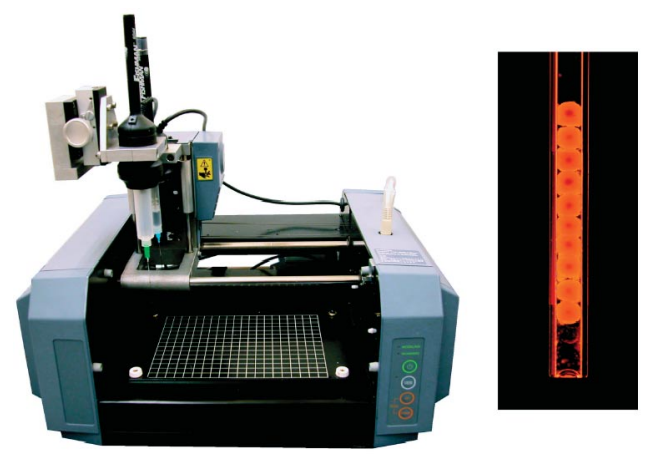

FIG. 1 (color). Special purpose 3D bioprinter with two mechanically driven extruders (left panel). One of the extruders hosts the bioink cartridge (right panel), a micropipette (the one shown has $500 \mu \mathrm{m}$ inner diameter) with the spherical aggregatebioink particles. The other extruder prints the biopaper-hydrogel scaffold. The $x-y$ stage and the $z$ directional motion of the extruders are fully computer controlled.

blue [25]; few dead cells were found in the center of the sheets). Note that the sheet is a 3D structure, its thickness being comparable to the aggregates' diameter.

In Fig. 3 we present simulation results for long tubular structures constructed via "layer-by-layer" deposition of rings of aggregates and supporting gel. Our motivation to consider tubes stems from the fact that they represent a fundamental unit of organ design (e.g., vasculature, lung, kidney, intestines) [26]. Three different arrangements, potentially with a hollow interior (i.e., lumen) and real biological relevance are shown. In Fig. 3(a), aggregates are made of a single cell type. Depending on the properties of the embedding gel (i.e., the value of $\gamma_{c g} / E_{T}$ ) the final configuration is either a tube or the system breaks up into solid spheres (reminiscent of the pearling instability in true

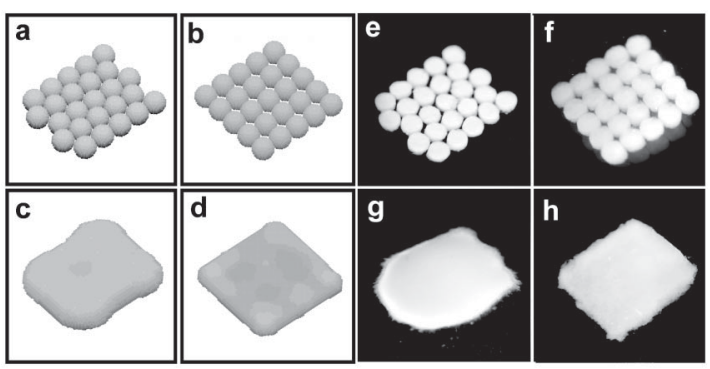

FIG. 2. Sheet formation depends on the initial configuration and the tissue-matrix interfacial tension. Two initial states, made of model cell aggregates, 925 cells each, packed in (a) a hexagonal and (b) a square lattice, after $250000 \mathrm{MCS}$ evolve into configurations shown in panels (c) $\left(\gamma_{c g} / E_{T}=0.8\right)$ and (d) $\left(\gamma_{c g} / E_{T}=1.4\right)$, respectively. For identical parameters, fusion from the hexagonal initial configuration is considerably faster. (e),(f) Similar structures of 25 aggregates of $\mathrm{CHO}$ cells (500 $\mu \mathrm{m}$ in diameter) were embedded in $1.0 \mathrm{mg} / \mathrm{ml}$ collagen type I. Compact sheets after $144 \mathrm{~h}$ of incubation are shown in panels (g) and (h). 


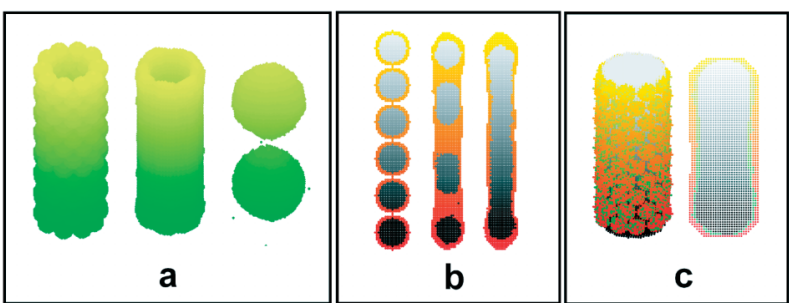

FIG. 3 (color). (a) Tube formation with model cells of the same type. In each ring, 10 aggregates, of 257 cells each, are placed contiguously along circles, and rings are closely packed in the vertical direction such that each aggregate touches two others from below and above. In a simulation, with $\gamma_{c g} / E_{T}=2$, after $2 \times 10^{5}$ MCS the result is the tube shown in the middle, whereas for $\gamma_{c g} / E_{T}=0.4$ the structure breaks up into two spheroids. (b) Spontaneous tube formation. The initial aggregates (left) are made of cells wrapping a gel core (index 2 below), embedded in a different type of gel (index 1 below). One composite aggregate includes 4169 lattice sites, part of which is occupied by cells, the rest by gel. The two final structures, in the middle and on the right, reached after $10^{5}$ MCS, were both simulated with $\gamma_{c g_{1}} / E_{T}=0.7, \gamma_{c g_{2}} / E_{T}=0.3$, and $\gamma_{\mathrm{g}_{1} \mathrm{~g}_{2}} / E_{T}=1.5$; the respective cell occupancies (of each aggregate's volume) were $66 \%$ and $50 \%$. (c) Tissue self-assembly in a 4-phase system made of two types of cells randomly intermixed in each aggregate (30\% type 2 , green) and two types of gel. The initial state (left) consists of 10 rings of 10 aggregates of 257 cells each. On the right side, an axial cross section of the final state, reached after $10^{5} \mathrm{MCS}$ is depicted. We associate indices 0 and 1 , respectively, with the embedding gel and the gel in the interior of the tube. Indices 2 and 3 designate, respectively, the green and red cell types. The interfacial energy parameters are $\gamma_{01} / E_{T}=1.8, \gamma_{02} / E_{T}=1.2, \gamma_{03} / E_{T}=0.7, \gamma_{12} / E_{T}=0.7$, $\gamma_{13} / E_{T}=1.2$, and $\gamma_{23} / E_{T}=0.4$.

liquids [27]). In Fig. 3(b), the initial aggregates may be thought of as prepared from cells of the same type, arranged on spheres made of a gel (different from the embedding gel). The final pattern depends on the properties of both gels and on the relative volume occupied by cells in the aggregates. (The energy of the system is now given in terms of three interfacial tensions.) Examples of tubular structures composed of only one type of cells are tubes in the mammalian lung, or small capillaries of the vasculature. In Fig. 3(c) the initial aggregates represent random mixtures of two cell types and the gel in the interior differs from the exterior one. This structure is described in terms of six interfacial tensions, which can be chosen to reach the final configuration on the right of Fig. 3(c). The kinetics of shape evolution is similar to that observed in sorting. The biological analogues of such structures are thick blood vessels, with endothelial cells lining their interior wall and smooth muscle cells (providing the contractile properties of the vessel), as well as extracellular matrix. To arrive at lumens, in the final tubular structures in Figs. 3(a)-3(c), the interior gel would be degraded (e.g., in case of collagen with the enzyme collagenase).
Fusion of aggregates, as shown and modeled in Figs. 2 and 3 , is the consequence of tissue liquidity. However, tissue liquidity implies that the final, equilibrium configurations should all correspond to a single sphere having the lowest interfacial energy. The sheets and tubes do not correspond to equilibrium states. However, with the suitable choice of parameters (here the properties of the embedding matrix) these structures can be made long-lived, as shown in Fig. 4. After a sharp decrease, the energy of the various model cellular patterns levels off (some faster than others), indicating that these structures are indeed changing slowly. Upon further evolution the energy eventually sharply decreases again, as in Fig. 4(d), due to changes in the construct's topology. However, once the desired structure is reached, the embedding gel can be removed, thereby preventing the further rearrangement of cells. The construct, if made of living cells, can subsequently be transferred and preserved in a bioreactor.

Our model represents a strong oversimplification of true tissue-extracellular matrix assemblies. In particular, cellmatrix interactions are dynamical: cells secrete new matrix materials and reorganize existing ones, which in turn provide the geometric architecture needed for cell movement [28]. Our Monte Carlo-based simulations cannot give account of such complicated processes. Neither can our experimental realization of cellular sheets be viewed as construction of organs. What our work implicates is that by providing initial cues to the biological system we can direct its self-assembly.

Our model operates with aggregates of identical particles, whereas true cellular spheroids (widely used for biological studies [29-34]) contain heterogeneous cell populations. It uses gross physical parameters, such as surface tension, cell-cell and cell-matrix couplings, whereas true biological interactions employ a myriad of molecular entities. The fact that despite these simplifications some of the model's predictions have experimentally been reproduced underlies the statement made at the be-
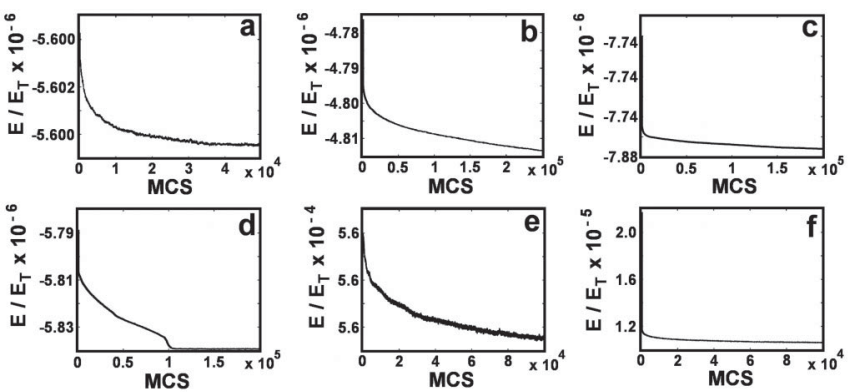

FIG. 4. The total interaction energy vs the number of MCS for the various simulations. (a) The single ringlike configuration (i.e., a one-layer tube; not shown). (b) The sheet simulation of Figs. 2(a) and 2(c). (c) The tube in the middle of Fig. 3(a). (d) The tube undergoing the pearlinglike instability on the right of Fig. 3(a). (e) The tube on the right of Fig. 3(b). (f) The longlived tube in Fig. 3(c). 
ginning: it is the universal physical mechanisms that are responsible for biological structure formation, which act on macroscopic (here supercellular-tissue) scale irrespective of the nature of interactions on the microscopic (here cellular and subcellular) scale. In particular, as far as structure formation is concerned, the details of how the $\varepsilon$ 's or $N_{c g}$ are determined by the genetic apparatus of the cell are immaterial. These quantities should be considered as effective interactions: $\varepsilon_{c c}, \varepsilon_{c g}$, and $\varepsilon_{g g}$ are determined, respectively, by the cell's adhesive apparatus (type of cell adhesion molecules, for example, cadherins [35,36] or members of the immunoglobulin family of cell adhesion molecules $[37,38])$, by the nature of cell-matrix adhesion (e.g., integrins [39]), and by the molecular composition of the matrix (type of matrix molecules, for example, fibronectin, collagen, tenascin [40]). The quantity $N_{c g}$ is related to the number of cell-cell adhesion or cell-substratum (i.e., matrix) molecules on the cell's surface, as well as the concentration of matrix molecules.

Our simple model provides biologically relevant and useful information. It confirms that biological structure formation depends on the scaffold that embeds the cellular material (a well-known fact in tissue engineering [41]). It suggests that it is a specific combination of cell-cell and cell-matrix interactions (i.e., $\gamma$ ) that eventually determines the final shape. It offers, in principle, a mechanism for optimizing structure evolution (by adjusting the value of $\gamma$ ). It implies that spherical cell aggregates composed of single or multiple cell types could be employed as bioink particles (respectively, monocolor or multicolor) and deposited by special bioprinters into biocompatible biopapers (a point demonstrated by printing specific arrangements of these bioink particles). Finally and most importantly, it points towards the possibility of employing physics-based knowledge on liquids to build physiologically relevant biological structures.

We are grateful to Roger Markwald for the use of the bioprinter. We have benefited from discussions with Ioan Kosztin and Vladimir Mironov. This work was supported by NSF (IBN-0083653; FIBR-0526854) and NASA (NAG2-1611).

[1] G. M. Whitesides and M. Boncheva, Proc. Natl. Acad. Sci. U.S.A. 99, 4769 (2002).

[2] J.D. Murray, Mathematical Biology (Springer, Berlin, 1993).

[3] S. A. Newman and W.D. Comper, Development 110, 1 (1990).

[4] M. S. Steinberg, Science 137, 762 (1962).

[5] M. S. Steinberg and T. J. Poole, Cell Behaviour, edited by R. Bellains, A.S.G. Curtis, and G. Dunn (Cambridge University Press, Cambridge, England, 1982).

[6] R. Foty, G. Forgacs, C. M. Pfleger, and M. S. Steinberg, Phys. Rev. Lett. 72, 2298 (1994).
[7] R. A. Foty, C. M. Pfleger, G. Forgacs, and M. S. Steinberg, Development 122, 1611 (1996).

[8] R. Gordon, N.S. Goel, M.S. Steinberg, and L. L. Wiseman, J. Theor. Biol. 37, 43 (1972).

[9] D. Beysens, G. Forgacs, and J. Glazier, Proc. Natl. Acad. Sci. U.S.A. 97, 9467 (2000).

[10] G. Forgacs, R. A. Foty, Y. Shafrir, and M. S. Steinberg, Biophys. J. 74, 2227 (1998).

[11] R. A. Foty and M.S. Steinberg, Cancer Res. 57, 5033 (1997).

[12] D. Duguay, R. A. Foty, and M. S. Steinberg, Dev. Biol. 253, 309 (2003).

[13] E. E. Robinson, K. M. Zazzali, S. A. Corbett, and R. A. Foty, J. Cell Sci. 116, 377 (2003).

[14] R. A. Foty and M. S. Steinberg, Dev. Biol. 278, 255 (2005).

[15] D. Godt and U. Tepass, Nature (London) 395, 387 (1998).

[16] A. González-Reyes and D. St. Johnston, Development 125, 2837 (1998).

[17] T. Hayashi and R. W. Carthew, Nature (London) 431, 647 (2004).

[18] F. Graner and J. A. Glazier, Phys. Rev. Lett. 69, 2013 (1992).

[19] J. A. Glazier and F. Graner, Phys. Rev. E 47, 2128 (1993).

[20] K. Jakab, A. Neagu, V. Mironov, R. R. Markwald, and G. Forgacs, Proc. Natl. Acad. Sci. U.S.A. 101, 2864 (2004).

[21] V. Mironov, R. Markwald, and G. Forgacs, Science and Medicine 9, 69 (2003).

[22] J. Israelachvili, Intermolecular \& Surface Forces (Academic Press, New York, 1997).

[23] N. Metropolis, A. W. Rosenbluth, M. N. Rosenbluth, A. H. Teller, and E. Teller, J. Chem. Phys. 21, 1087 (1953).

[24] J.C. M. Mombach, J.A. Glazier, R.C. Raphael, and M. Zajac, Phys. Rev. Lett. 75, 2244 (1995).

[25] I. Mickuviene, V. Kirveilene, and B. Juodka, Toxicol. In Vitro 18, 639 (2004)

[26] B. Lubarsky and M. A. Krasnow, Cell 112, 19 (2003).

[27] L. Rayleigh, Philos. Mag. 34, 145 (1892).

[28] R. K. Sawhney and J. Howard, J. Cell Biol. 157, 1083 (2002).

[29] P. G. Layer and A. Robitzki, A. Rothermel, and E. Willbold, Trends Neurosci. 25, 131 (2002).

[30] R. Glicklis, J.C. Merchuk, and S. Cohen, Biotechnol. Bioeng. 86, 672 (2004).

[31] T. Korff, S. Kimmina, G. Martiny-Baron, and H.C. Augustin, FASEB J. 15, 447 (2001).

[32] R. M. Sutherland, Science 240, 177 (1988).

[33] J. M. Kelm, N. E. Timmins, C. J. Brown, M. Fussenegger, and L. K. Nielsen, Biotechnol. Bioeng. 83, 173 (2003).

[34] L. J. Kaufman, C. P. Brangwynne, K. E. Kasza, E. Filippidi, C.D. Gordon, T.S. Deisboeck, and D. A. Weitz, Biophys. J. 89, 635 (2005).

[35] M. Takeichi, Development 102, 639 (1988).

[36] B. M. Gumbiner, J. Cell Biol. 148, 399 (2000).

[37] A. F. Williams and A. N. Barclay, Annu. Rev. Immunol. 6, 381 (1988).

[38] T. Hunkapiller and L. Hood, Adv. Immunol. 44, 1 (1989).

[39] R. O. Hynes, Cell 48, 549 (1987).

[40] B. Alberts, Molecular Biology of the Cell (Garland Science, New York, 2002).

[41] L. G. Griffith and G. Naughton, Science 295, 1009 (2002). 